\title{
Magnetic Force Microscopy of Brain Microtubules
}

\author{
Pavlo Mikheenko \\ Department of Physics \\ University of Oslo \\ Oslo, Norway \\ pavlo.mikheenko@fys.uio.no
}

\begin{abstract}
Being biological object, microtubules attract significant attention in physics, since it is believed that they are responsible for quantum processing of information in the brain. There were, however, no direct experiments checking such a statement. Recently, strong advancement in quantum computing took place utilizing properties of superconductors at low temperatures. Following this progress, it was proposed that brain microtubules are superconducting at room temperature allowing quantum processing of information. Moreover, the evidence of room-temperature superconductivity in brain slices containing microtubules was obtained by electrical transport measurements, and even specific scenario of quantum processing in the microtubules has been suggested. These results, however, are not yet accepted by the scientific community as there are no known attempts to reproduce them. Another step in proving superconductivity would be confirming ideal diamagnetism of microtubules, since ideal diamagnetism is more fundamental property of superconductivity than perfect conductivity, some features of which were seen indirectly, or the existence of energy gap, which was already confirmed by the transport measurements. Here brain microtubules are examined by the magnetic force microscopy. The evidence of strong diamagnetism and its sensitivity to the water content in the microtubules is obtained. This gives another strong argument in favor of the concept suggesting superconductivity-based quantum processing of information in living organisms.
\end{abstract}

Keywords - brain, microtubules, magnetic force microscopy, superconductivity, quantum processing of information.

\section{INTRODUCTION}

Microtubules are quasi one-dimensional tubes of the diameter of $25 \mathrm{~nm}$ made of alpha and beta tubulin proteins. They are ubiquitous in living organisms, form scaffolding of eukaryotic cells, can be as long as 50 micrometers and are of primary importance in nerve cells [1,2]. Additionally to biology, microtubules are objects of significant attention in physics following suggestion that they might be responsible for quantum processing of information in the brain [3]. In [3], processing of information was suggested to take place utilizing quantum states of tubulin proteins. The theory was criticized as unrealistic at room temperature [4]. There is, however, another possibility to have quantum processing in microtubules, which is in line with recent advances of practical quantum computing that utilizes properties of superconductors. This possibility is based on the assumption that microtubules are superconducting at room temperature. Ideas about superconductivity in brain were formulated as early as in 1972 [5], and theoretically, using electronelectron interaction, it is possible to achieve superconducting state in quasi one-dimensional organic chains with critical temperature $\left(\mathrm{T}_{\mathrm{c}}\right)$ far above room temperature, as it was found by Little [6].
There already presented electrical transport measurements indicating existence of superconductivity in brain slices [7], and even specific mechanism for quantum processing in microtubules is suggested [8]. In [7], currentvoltage characteristics were obtained at room temperature, which resemble current-voltage characteristics of superconductors, and, more important, superconducting energy gap was measured allowing estimating $\mathrm{T}_{\mathrm{c}}$, which appeared to be close to that predicted by Little [6]. However, these measurements are still not reproduced in other laboratories and although interest to the activity is reasonably high, the ideas are not accepted yet by the scientific community. Obviously, more evidences of superconductivity in the microtubules are necessary.

To be efficient in quantum processing of information at ambient conditions, similar to what is achieved in modern quantum computers at low temperatures [9], $\mathrm{T}_{\mathrm{c}}$ of superconductor should be much higher than room temperature [8]. By introducing electron-electron interaction between the molecular complexes and conducting quasi onedimensional molecular chains, $\mathrm{T}_{\mathrm{c}}$ of $\approx 2200 \mathrm{~K}$ has been predicted [6]. This is close to what was found in brain slices [7], and it is sufficient for enabling quantum tunneling regime at room temperature $[8,10]$.

In [7], microtubules were treated statistically, as main component of the brain tissue. Here, isolation of microtubules and their bundles, and the measurements of their magnetic properties on nanometer scale have been done. Investigating magnetic properties of a material, which is expected to be superconductor is important, as ideal diamagnetism is more fundamental property of superconductivity than perfect conductivity.

Since diameter of microtubules is very small (about 25 $\mathrm{nm}$ ), a magnetic scanning technique should be applied at this length scale too. This is in the operational range of magnetic force microscopy (MFM) [11-15]. However, such a technique requires special preparation of the samples allowing exposing microtubules, their bundles or fragments of nerve cells on a flat nanometer-smooth surface.

\section{EXPERIMENTAL METHODS}

An original technique has been developed for the preparation of samples. It starts with pig brain tissue, the same as was used in [7], delivered from the food chain and fixed in standard biological solution (4\% Paraformaldehyde $+2.5 \%$ glutaraldehyde in $\mathrm{Na}$ Cacodylate $0.1 \mathrm{M}$ buffer). The technique consists of fragmenting brain tissue with a blade and then repetitive multiple rinsing of it with the aqueous solution of graphene nano-plates. The graphene nano-plates break the tissue fragments, separate parts of cells and microtubules and suspend them in the liquid. The saturated 
liquid was dropped on a nanometer-smooth glass substrate and dried in a flow of air. The solidified drop provides a smooth surface with microtubules and fragments of nerve cells on top of it. The substrate was set in the scanning probe microscope JPK NanoWizard 4.0 above a permanent magnet of the strength of $0.58 \mathrm{~T}$. The MFM study has been carried out in alternating current (AC) mode at a resonance frequency of about $74 \mathrm{kHz}$, The NANOSENSORS (TM) PPP-MFMR AFM magnetic probes have been used for the magnetic imaging. The measurements has been done in external magnetic field of permanent magnet. Several signals have been recorded in the MFM imaging, among them amplitude and phase shift of AC oscillations.

At low distances between the probe and the surface of the sample, both van der Waals and magnetic forces act to the probe. To separate these forces, a standard procedure of raising the probe to the height, at which van der Waals force becomes negligible, was used. This procedure is known as the two-pass scanning technique $[12,13]$. In the first pass, the probe measures the surface topography. In the second pass, it follows the topography at a greater height.

In the technique, magnetic properties are extracted from the phase shift maps, which provide information about the gradient of force acting along the axis perpendicular to the surface of the sample $[14,16,17]$ :

$$
\Delta \varphi=\mathrm{A} \partial \mathrm{F} / \partial \mathrm{z}
$$

In (1), $\Delta \varphi$ is the phase shift, $\mathrm{F}$ is the force, $\mathrm{z}$ is the coordinate along the axis perpendicular to the surface and $\mathrm{A}$ is a negative constant. At large heights above the surface, typically above $20 \mathrm{~nm}$ [18], the maps give pure information about the magnetic interaction. Above the areas of enhanced magnetization with the same direction as the magnetization of the probe, the gradient of the magnetic force is positive, and being measured and converted in the intensity of color, these areas look darker than the background [18]. On the contrary, diamagnetic spots should look brighter.

At small heights above the surface of few nanometers, the situation is more complicated. There the short-range attractive van der Waals force with positive force gradient changes, at smaller $\mathrm{z}$, to the repulsive force with negative gradient, which sets into action due to Pauli exclusion principle. Correspondingly, with the increase of $z$, the color in phase shift map changes from bright to dark, decaying in intensity with increase of $z$. The areas of enhanced magnetization display dark color up to very large $\mathrm{z}$ [18], while seeing the transformation of the color from dark to bright at higher $\mathrm{z}$ would mean registering diamagnetism.

\section{RESULTS AND DISCUSSION}

The individual microtubules, their bundles and fragments of nerve cells were identified in phase shift maps shortly after the preparation of a sample and its imaging by the MFM. An example of individual microtubules is shown in Fig. 1. They are seen as bright lines in a) and as bright dots surrounded by dark areas in the better-resolved image b), which is fragment of a) surrounded by the red lines in the plot. Both maps were recorded at height of $5 \mathrm{~nm}$ above the surface.

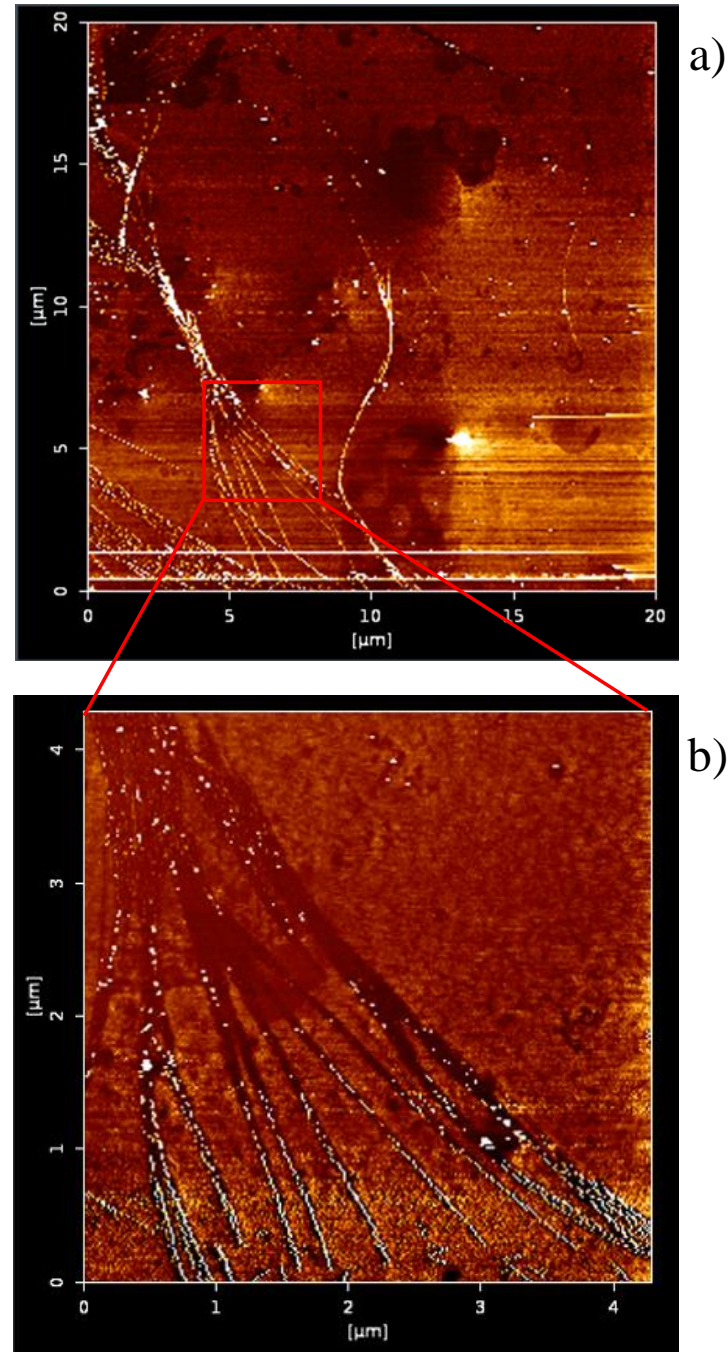

Fig.1 a) Phase shift map of a solidified drop containing individual microtubules and their bundles (bright lines) recorded at height of $5 \mathrm{~nm}$ above the surface. b) Fragment of the image a) marked by the red lines in a). Both images are recorded at MFM set point of $0.3 \mathrm{~V}$.

Some of the microtubules in Fig. 1 are united in the bundles. Few hours after the preparation, however, even in spite of being fixed in special solution, individual microtubules begin depolymerize. The start of this process is seen at the bottom of Fig. 1b, where some of them look broken. The bundles with microtubules linked together appear to be more stable and can be imaged for several days.

Since the images in Fig. 1 were recorded at low height, they mainly represent attractive van der Waals forces (dark color). The bright color corresponds to mixed-in repulsion due to Pauli exclusion principle. The action of Pauli principle can be offset by regulating the strength, with which probe interacts with the sample. It is done by changing MFM set point (SP) in the settings of the scan. The images in Fig. 1 were recorded at SP of 0.3 V. For a larger SP, probe stops at bigger distance (on fractions of nanometer) from the surface, and the bright spots could be removed from the dark areas, as shown in Fig. 2, in which images are recorded at SP of $0.7 \mathrm{~V}$. Most important, however, what would happen if the second pass is recorded (with the same SP) at bigger than $5 \mathrm{~nm}$ height above the surface. 

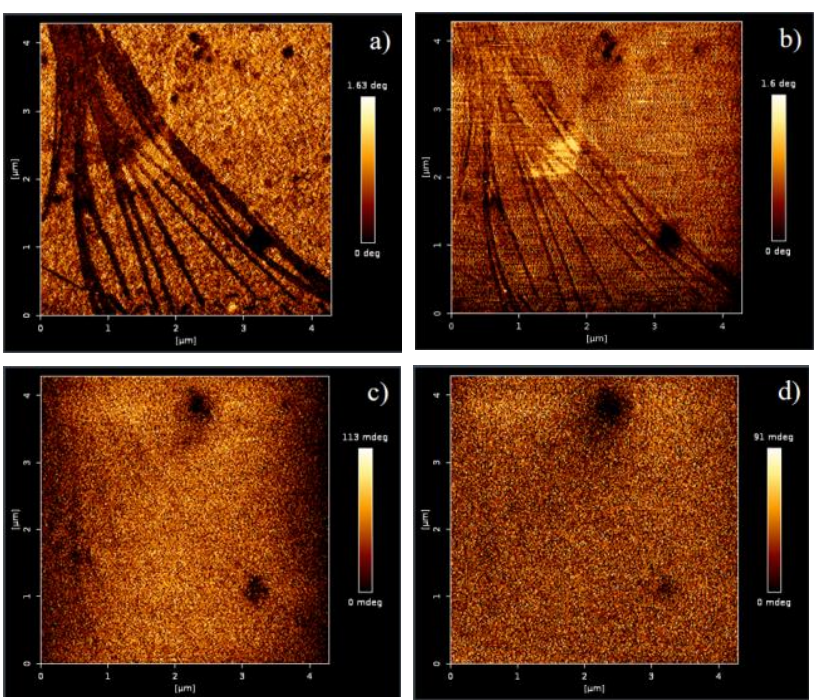

Fig.2 Phase shift maps of a bundle of microtubules as in Fig. 1 recorded at MFM set point of $0.7 \mathrm{~V}$ and heights of a) 0, b) $5 \mathrm{~nm}$, c) $12 \mathrm{~nm}$ and d) $15 \mathrm{~nm}$ above the surface.

The phase shift maps of the same bundle of microtubules as in Fig. 1, recorded at heights of a) 0, b) $5 \mathrm{~nm}$, c) $12 \mathrm{~nm}$ and d) $15 \mathrm{~nm}$ above the surface with SP of $0.7 \mathrm{~V}$ are shown in Fig.2. It is clear that image of microtubules vanishes at height of about $15 \mathrm{~nm}$, at which van der Waals force stop acting to the probe. Although some weak bright traces from microtubules could be seen in d), the magnetism is not conclusive there [18]. Stopping study at this point would lead to conclusion that microtubules are non-magnetic. Indeed, with accuracy of 0.091 degree in the phase shift, as it is seen from the scale bar in d), which is more than two orders of magnitude smaller than expected response for ideal diamagnet [19], there is no evident magnetism here.

The traces of bright color in Fig. 2d, however, encouraged to look for diamagnetic response in other areas of the sample and, indeed, it was found in the bundles of microtubules, as shown in Fig. 3. Although diamagnetism is still not strong (see scale bars on the right), it is clearly present here [18] and lasts up to height of at least $50 \mathrm{~nm}$.
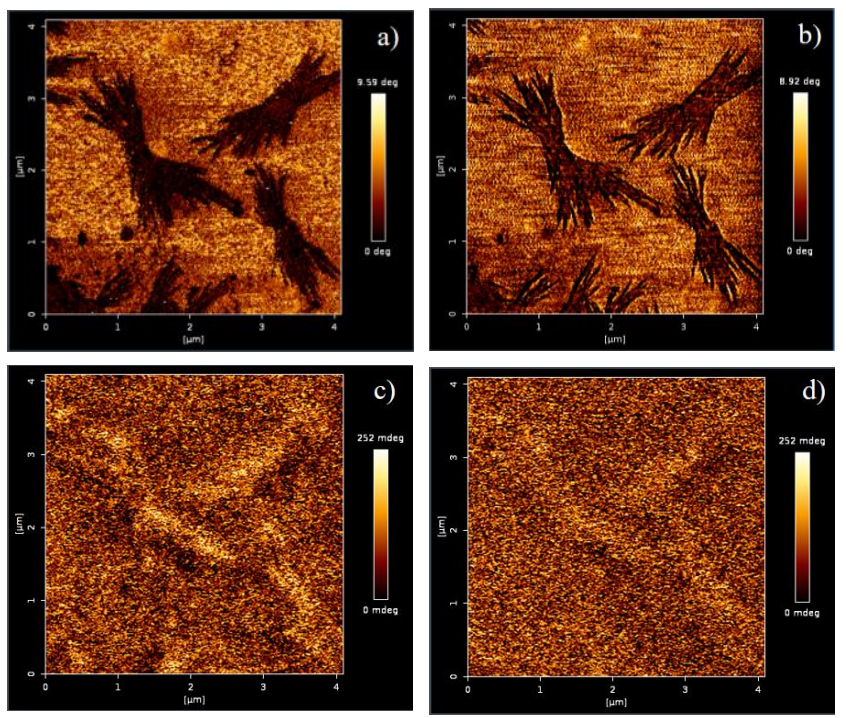

Fig.3 Phase shift maps of the bundle of microtubules recorded at heights of a) 0, b) $5 \mathrm{~nm}$, c) $30 \mathrm{~nm}$ and d) $50 \mathrm{~nm}$ above the surface.
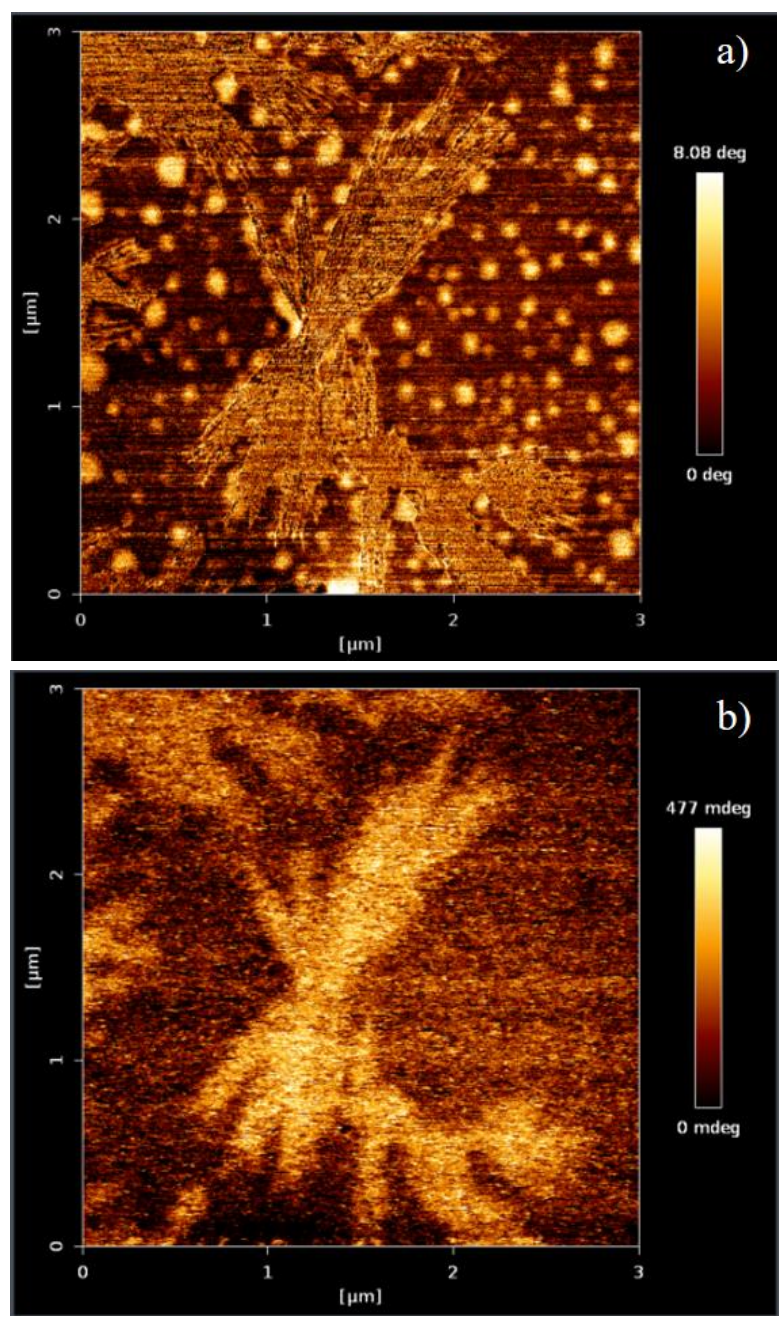

Fig.4 Phase shift maps of a bundle of microtubules after exposure to water vapor recorded at heights of a) 0 and b) $35 \mathrm{~nm}$. The nano-drops of water are seen in a).

There is good reason why diamagnetism is not strong in Figs. 2 and 3. This reason is dehydration. The importance of water for coherent and superconducting behavior of microtubules was previously emphasizes in [7] and [20]. Following this knowledge, the sample was exposed to water vapor. The result was strong increase in diamagnetism even straight after the exposure, as shown in Fig. 4. The images in this figure are recorded at heights of a) 0 and b) $35 \mathrm{~nm}$. The image in b) is outside the range of van der Waals forces, which could be detected by this technique, and is of magnetic origin. The diamagnetic response in the image is strongly increased comparable to that in Figs. 2 and 3.

The nano-drops of water are also seen in Fig. 4a, but although water is diamagnetic, no traces of it are present in Fig. 4b. It is because diamagnetic susceptibility of water $\left(\chi_{\mathrm{v}}\right)$ is very low: $-9.05 \times 10^{-6}$, which is about five orders of magnitude lower than susceptibility of ideal diamagnet or superconductor $\left(\chi_{v}=-1\right)$. It is far outside of the range of detection by this technique. The diamagnetic signal in Fig. 4 is not the largest obtained in the MFM experiments with the brain tissue. By repeating saturation by water vapor several times, diamagnetic response have been achieved with $\chi_{\mathrm{v}}$ close to -1 . One of the examples of the phase shift maps for such strong diamagnetism is shown in Fig. 5. 


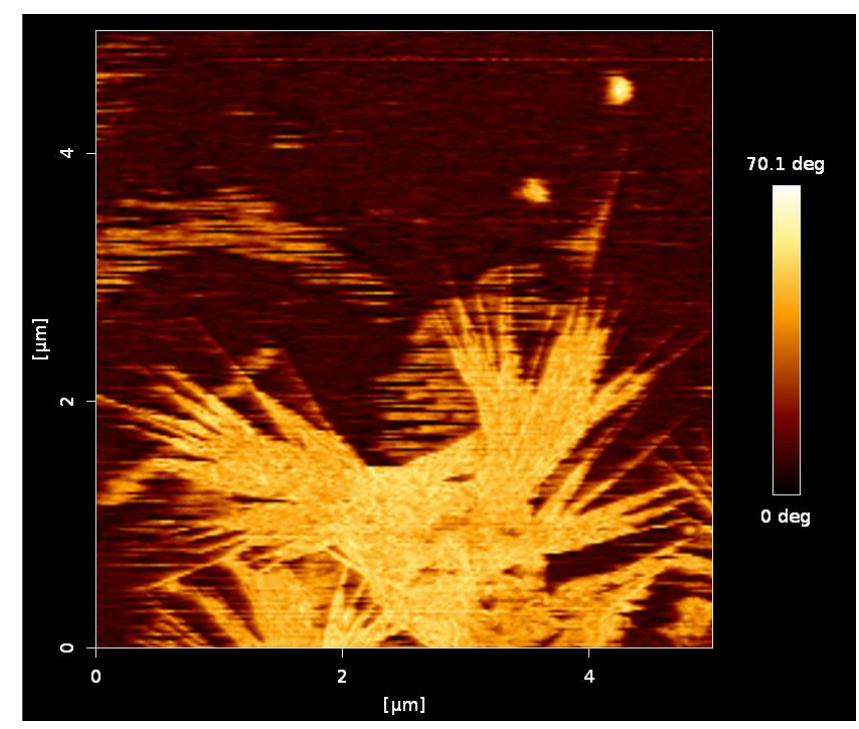

Fig.5 Phase shift map for a bundle of microtubules after multiple exposure to water vapor recorded at height of $20 \mathrm{~nm}$.

The image in Fig. 5 is recorded at the height of $20 \mathrm{~nm}$, which is outside of the range of van der Waals force (compare with Fig. 1). The top value in the scale bar in this image is 70.1 degrees in comparison with fractions of a degree (mdeg) in other images. The details of the calibration procedure and prove of ideal diamagnetism will be reported elsewhere [19].

Overall, additionally to evidences from the transport measurements [7] and the observation of the coherent behavior in the water-filled microtubules [20], MFM study gives another strong argument supporting the idea of robust room temperature superconductivity in water-saturated brain microtubules. As the microtubules are present not only in the brain, but also in every cell of advanced living organisms, these organisms could be regarded as coherent superconducting systems. In its turn, superconductivity with $\mathrm{T}_{\mathrm{c}}$ far above room temperature [7] provides basis for quantum processing of information [8].

\section{CONCLUSION}

Magnetic force microscopy study of pig brain microtubules has been carried out revealing strong diamagnetism and giving, additionally to transport measurements, another evidence of superconductivity in the nerve cells. Special graphene-based technique allowing exposing microtubules and their bundles on nanometersmooth surfaces has been developed to carry out this study. It was found that diamagnetism is very sensitive to water content in the microtubules. The bundles of microtubules fixed in a standard formalin-based solution allow multiple re-saturation by water returning to the state of strong diamagnetism. Accepting concept of superconductivity in microtubules would resolve is a simple way basic problems related to quantum processing of information in living organisms.

\section{REFERENCES}

[1] A. Akhmanova, and M. O. Steinmetz, "Tracking the ends: a dynamic protein network controls the fate of microtubule tips," Nature Reviews Molecular Cell Biology vol. 9, 309, April 2008.

[2] M. Lasser, J. Tiber, and L. A. Lowery, "The role of the microtubule cytoskeleton in neurodevelopmental disorders," Front. Cell. Neurosci., vol. 12, 65, June 2018.

[3] S. Hameroff, "Quantum computation in brain microtubules? The Penrose-Hameroff 'Orch OR' model of consciousness," Phil. Trans. R. Soc. A, vol. 356, pp. 1869-1896, August 1998.

[4] E. C. Behrman, K. Gaddam, J.E. Steck, and S.R. Skinner, "Microtubules as a Quantum Hopfield Network," In The Emerging Physics Of Consciousness, Jack A. Tuszynski Ed. Berlin Heidelberg: Springer-Verlag, 2006, pp. 351-370.

[5] E. H. Halperin and A. A. Wolf, "Speculations of superconductivity in biological and organic systems," In: Advances in cryogenic engineering, vol. 17, K.D. Timmerhaus, Ed. New York: Springer Science + Business Media, 1972, pp 109-115.

[6] W. A. Little, "Possibility of synthesizing an organic superconductor," Phys. Rev., vol. 134, pp. 1416-1424, June 1964.

[7] P. Mikheenko, "Possible superconductivity in the brain," J. Supercond. Novel Magn., vol. 32, pp. 1121-1134, May 2019.

[8] P. Mikheenko, "Nano superconductivity and quantum processing of information in living organisms," 2020 IEEE 10th International Conference Nanomaterials: Applications \& Properties (NAP), Sumy, Ukraine, 2020, pp. 02SNS02-1 - 02SNS02-4.

[9] M. H. Devoret, and R. J. Schoelkopf, "Superconducting circuits for quantum information: an outlook," Science vol. 339, pp. 1169-1174, March 2013.

[10] M. Lyatti, M. A. Wolf, I. Gundareva, M. Kruth, S. Ferrari, R. E. Dunin-Borkowski, and C. Schuck, "Energy-level quantization and single-photon control of phase slips in $\mathrm{YBa}_{2} \mathrm{Cu}_{3} \mathrm{O}_{7-\mathrm{x}}$ nanowires," Nat. Commun., vol. 11, 763, Feb. 2020.

[11] A. de Lozanne, "Application of magnetic force microscopy in nanomaterials characterization," Microsc. Res. Tech., vol. 69, pp. 550-562, May 2006.

[12] S. Schreiber, M. Savla, D. V. Pelekhov, D. F. Iscru, C. Selcu, P. C. Hammel, and G. Agarwal, "Magnetic force microscopy of superparamagnetic nanoparticles," Small, vol. 4, pp. 270 - 278, Feb. 2008.

[13] C. S. Neves, P. Quaresma, P. V. Baptista, P. A. Carvalho and J. P. Araújo, "New insights into the use of magnetic force microscopy to discriminate between magnetic and nonmagnetic nanoparticles," Nanotechnology, vol. 21, 305706, July 2010.

[14] D. Passeri, C. Dong, M. Reggente, L. Angeloni, M.o Barteri, F. A. Scaramuzzo, F. De Angelis, F. Marinelli, F. Antonelli, F. Rinaldi, C. Marianecci, M. Carafa, A. Sorbo, D. Sordi, I. WCE Arends, and M. Rossi, "Magnetic force microscopy. Quantitative issues in biomaterials," Biomatter, vol. 4, e29507, July 2014.

[15] G. Cordova, B. Y. Lee, and Z. Leonenko, "Magnetic Force Microscopy for Nanoparticle Characterization," NanoWorld J., vol. 2, pp. 10-14, April 2016.

[16] B. Torre, G. Bertoni, D. Fragouli, A. Falqui, M. Salerno, A. Diaspro, R. Cingolani, and A. Athanassiou, "Magnetic, force microscopy and energy loss imaging of superparamagnetic iron oxide nanoparticles," Sci. Rep., vol. 1, 202, Dec. 2011.

[17] D. Sarid, Scanning Force Microscopy, New York, U.S.A.: Oxford University Press, 1994.

[18] A. L. Campaña, N. Joudeh, H. Høyer, A. Røyne, D. Linke, and P. Mikheenko, "Probing van der Waals and magnetic forces in bacteria with magnetic nanoparticles," 2020 IEEE 10th International Conference Nanomaterials: Applications \& Properties (NAP), Sumy, Ukraine, 2020, pp. 01NSSA03-1-01NSSA03-5.

[19] P. Mikheenko, "Ideal diamagnetism in brain microtubules," unpublished.

[20] S. Sahu, S. Ghosh, B. Ghosh, K. Aswani, K. Hirata, D. Fujita, and A. Bandyopadhyay, "Atomic water channel controlling remarkable properties of a single brain microtubule: Correlating single protein to its supramolecular assembly," Biosens. Bioelectron., vol. 47, pp. 141148, Sept 2013. 